\title{
Allvar Gullstrand †
}

Am 28. VII. 1930 ist in seinem 68. Lebensjahre der schwedische Augenarzt Allvar Gullstrand gestorben. Ein Leben unermüdlicher und im höchsten Maß erfolgreicher Forscherarbeit ist zu Ende gegangen. Die Wissenschaft hat einen Verlust erlitten, wie sie ihn etwa bei dem Tocle Hermanns von Helmholtz zu beklagen hatte. Nicht nur unser engeres Fach der Augenheilkunde, sondern viele weitere Gebiete, die Physik in erster Linie uncl die technische Optik, die Physiologie und Mathematik sincl betroffen worden. Alle verdanken ihm wertvolle Bereicherung und För-derung.

Soil an dieser Stelle ein Überblick über dieses Arbeitsleben gegeben werden, so sei seines Wirkens in der Augenheilkunde in erster Linie ge-dacht. 1888 approbiert erwarb Gullstrand 1890 den Doktorgrad und wurde Allvar Gullstrand $\uparrow$.

327

189 ! in Stockholm Privatdozent fur Augenheilkunde. Das Jahr 1894 führte ihn nach Upsala. Dort wirkte er zuerst als außerordentlicher Professor und von 1904 ab als Vorstand der augenärzilichen Abteilung der Uni-versitätskliniken, bis ihm 1914 eine persönliche Professur für physio-logische und physikalische Optik übertragen wurde, die. ihn von der Ver-pflichtung zu klinischer und Lehrtätigkeit entband. Die Ricbtung seiner wissenschaftlichen Neigung gibt sich sogleich in seiner ersten Arbeit zu erkennen. Sie gilt der Verwendung des Ophthalmometers von /aval und $<$ Sckiöt $\sum$ zur Messung des Astigmatismus mit Hilfe des Querfehlers. der Denivellation, in einer einzigen Einstellung. Für die Ermittlung der Krümmung der Seitenteile der brechenden Flächen, zunächst wiecler der Hornhaut, macht er sich später unabhängig von cliesem Gerät, indem er ein photographisches Verfahren ausbildet. Die Formelableitung wird verein-facht clurch die Einführung der Kehrwerte der Längen, der Konvergenzen, in die Rechnung. fiber die gleichzeitige Bestimmung des Brechungs-zustandes und der Sehschärfe hancl-elt er in einer erschöpfenden Arbeit. Darin werden Unt $<$ ersucbungen der Sehschärfe und der Vergrößerung durchgeführt und eine strenge Begriffsbestimmung gegeben. Auf der dabei gewonnenen Grundlage erläutert er dann die Optometeranlagen und setzt auch den Plan eines eigenen, von ihm (seit 1888) gebrauchten Gerätes auseinander. Wenn Gullstrand nach einer so eingehenden Beschäftigung mit diesen í"ragen gleichwohl dem alten Dondersischen Verfahren den Vorzug gibt, so können die dort angegebenen Grüncle wohl auch heute noch als eine Warnung vor der Überschätzung derartiger optometrischer Vorrichtungen gelten. Diese Arbeit, die ursprünglich in schwedischer Sprache erschienen ist, wurde wie zwei andere, eine über einen deutlich pulsierenden Hornhautkegel uncl eine über einen hinteren Linsenkegel, 1922 ins Deutsche übertragen. Seine wichtigen großen Arbeiten hat Gull-slrand sogleich in deutscher Sprache veröffentlicht. Daß sie ihm selbst ganz zu Gebot stand, clavon haben sich die Fachgenossen auch gelegentlich der Aussprachen in den Versammlungen der Deutschen Ophthalmologischen Gesellschaft, die er fast regelmäßig besuchte, überzeugen $1 \ll J n n e n$. Am weitaus bekanntesten von alien Früchten seiner Arbeit dürfte wohl die Blendenlampe sein. Dieses Gerät, das als Spaltlampe heute eine fast unentbehrliche Hilfe in der Sprechstunde des 
Augenarztes geworden ist, war von ihm - und das ist bemerkenswert genug - ursprünglich für seine ophthalmometrische Forschungsarbeit gebaut worden. Es hat sich gewisser-maßen nebenbei als ein ungemein vorteilhaftes Beleuchtungsgerät für die täglichen Untersuchungen erwiesen, da es die längst vorhandenen ver-größcrnden Beobachtungsgeräte erst zu voller Wirksamkeit gebracht hat und uns so einen ungeahnt tiefen Einblick in die Feinheiten des Gewebs-aufbaues des Auges gewährt. War hiermit die fokale Beleuchtung der Augengewebe auf das vollkommenste durchgeführt, so bildet das gleiche Gerät einen wesentlichen Bestandteil der großen Gullstrandschen Augen-spiegel. Gait die Arbeit des Verstorbenen in allererster Linie der Er-kenntnis, so fehlt seine Einwirkung auf die Behandlung keineswegs. Es ist allbekannt, daß die mit Hilfe einer asphärischen Fläche vom Astigmatismus \&chiefer Bündel befreiten

Starbrillengläser seinen Namen tragen. Dadurch wollte M. von Rohr an eine wertvolle Förderung 328

Allvar Gullstrand $\dagger$.

crinnern, die bei der Aufnahme der Arbeit an der Brille in Jena auf ihn wirkte. Stand doch damals die Kenntnis von der Bedeutung des Augenclrehpunktes für die Planting der Brille ohne weiteres zur Ver-fügung; denn Gullstrand hatte einige Jahre vorher, als er von dem Zeissischen Werk eine Lupe schwacher Vergrößerung wünschte, ausdrück-lich angegeben, daß sie für den Augendrehpunkt als Blcndenort vom Astigmatismus schiefer Bündel und von Verzeichnung befreit sein sollte. Es war die Verantlinse. Von rein klinischen Arbeiten ist außer den oben angeführten Arbeiten bier seine Operation zur Neubildung eines Bindehaut-sackes und sein o·bjektives photographisches Verfahren zur Diagnose der Augenmuskellähmungen zu nennen. Der klinische Ophthalmologe, als der Gullstrand bis 1914 tätig war, ist nach seinen eigenen Worten nur seinen persönlichen Schülern bekannt. Darüber wird sich niemand wundern, der seine ungeheuere Forscherarbeit auf dem Gebiet der Physiologie und der Optik auch nur obenhin betrachtet.

Seine Tätigkeit in der Augenheilkunde hatte Gullstrand vox Aufgaben gestel.lt, die in der erschöpfenden Weise, wie er sie erstrebte, mit den damals vorhandenen Hilfsmitteln nicht gelí > st werden konnten, und zu deren Lösung auch die nötigen mathematischen Werkzeuge keineswegs griffbereit vorlagen. Gullstrand hat dazu noch unbekanntes Gebiet erst selbst erforschen müssen.

Er schlug, bei seiner Aufgabe vom Menschenauge ausgehend, zur Erforschung der optischen Abbildung von vornherein grundsätzlich cinen anderen Weg ein, als ihn die Wissenschaft, bestimmt durch die Bedürf-nisse der technischen Optik, bisher verfolgt hatte. Man betrachtete $\notin$ lort den Abbildungsvorgang unter den L $\hat{I}<2 \ddot{A} /$ ? ischen Voraussetzungen, d. h. unter gewissen einschränkenden, bei der Anwendung optischer Geräte nic zu verwirklichenden Bedingungen, indem man einen unendlich dünnen Raum um die Achse ausgerichteter Umdrehungsflächen und gleichmäßig brc-chende Mittel annimmt. Für diesen Fall gelangte man zu einfachen Beziehungen zwischen Ding- und Bildr»um, indem einem jeden Punkt bzw. einer jeden Linie des Dingraumes ein Punkt bzw. eine Linie des Bild-raumes zugeordnet war. Sobald man in der Wirklichkeit, eben bei dem Gebrauch optischer Geräte, diesen Raum überschritt, vermißte man diese wünschenswerten Beziehungen, fand Abweichungen und bezeichnete sie, die Wirklichkeit, als Fehler. Man suchte hauptsächlich im Wege der Reihenentwicklung nach ' den Mitteln, diese Fehler zu beseitigen oder wenigstens möglichst klein zu machen. Im Gegensatz hierzu untersuchte Gullstrand die tatsächlichen Verhältnisse, und zwar vom allgemeinen Fall ausgehend. Er verwendete dazu eigene Mittel. Da für die optische Abbildung die Strahlenvereinigung und die optische Projektion eine maß-gebende Rolle spielen, so erforschte 
er zunächst den Aufbau der von einem Punkt ausgehenden oder auf einen solchen gerichteten, an einer stetig gekrümmten Fläche gebrochenen Bündel. In jedem Punkt eines solchen gibt es eine Fläche, die auf alien Strahlen senkrecbt steht, eine Wellenfläche. Die Lichtstrahlen sind dann Lote auf dieser Fläche. Die Gesamtheit der Lote, die durch irgendeine Hauptkrümmungslinie der Fläche hindurchgeht, bildet eine einfache übersehbare Fläche, aus clere $\pi$ Eigenschaft sich die Entscheidung darüber ableiten läßt, ob ein 1)eliebig Allvar Gullstrand †.

329

ausgewählter Strahl (Leitstrahl) von einem und mehreren nächstliegenden geschnittcn wird, und wo die Schnittpunkte liegen, wenn man von einem ausgewählten Strahl zu anderen und anderen übergeht. Die Untersuchung hat Gullstrand mit Hilfe der Flächentheorie durchgeführt. In der Rech-nung ausgehend von der allgemeinen Flächengleichung. Als erste Stufe dieser Entwicklung erhielt er die Gesetze des Astigmatismus. Sie gab Gull-strand zum ersten Mai vollständig an, u. a. zeigend, daß im dünnen astig-matischen Bündel keineswegs, wie Sturm meinte, die sämtlichen Strahlen durch zwei Brennlinien hindurchgehen, sondern daß tatsächlich nur zwei engste Einschnürungen im Bündel vorhanden sind. In den hòhereii Stufen der Entwicklung werden weiterhin die Erscheinungen im Bündelaufbau vollständig klargelegt, von denen man die Koma und die Abweichung wegen der Kugelgestalt der brechenden Flächen schon früher auf anderem Wege kennengelernt hatte. Will man die Frage der optischen Projektion andeuten, so kann mit Gullstrand an den Versuch erinnert werden, bei dem ein undurchsichtiges gerades Stäbchen in einer zum Leitstrahl eines homozentrischen Bündels senkrechten Ebene angeordnet und nach der Brechung sein Schatten auf einer ihr parallelen Schirmebene aufgefangen wird. Es set nur erwähni, daß die Schattenlinie der Schatten gebenden durchaus nicht ähnlich zu sein braucht. Bei der Untersuchung der Abbildungsvorgänge hat G. nur das Brechungsgesetz und die gerad-linige Fortpflanzung des Lichtes vorausgesetzt. Daraus hat er die allgemeinen Abbilclungsgesetze abgeleitet und in $\mathbf{m} \mathbf{m}$ jahrelanger Arbeit zu höheren Stufen weitergeführt. Die Gesetze wurden unter geeigneten Vor-aussetzungen so zusammengefaßt, daß die Abbildung nicht auf die ein-zelnen Flächen einer Folge bezogen zu werden brauchten, sondern daß sie durch die Aufnahme der Systemkonstanten ausgesprochen werden kon $\pi$ ten. Die erste Stufe solcher Systemgesetze ist schon durch Gauß ermittelt worden. $\mathrm{Da} ß$ die Gaußische Abbildung nicht immer in der Strenge ihrer Voraussetzung verstanden wird, hat Gullstrand nicht unterlassen zu rügen. Wiederholt betont er, daß es außerhalb der Achse einer ausgerichteten Folge überhaupt keine allgemeine punktuelle Abbildung gibt, ebensowenig eine streng kollineare optische Abbildung. Wohl könne man den einen Raum zu einem anderen mathematisch so in Beziehung bringen, daß ein Punkt des einen einem Punkt des anderen, eine Linie des einen einer solchen des anderen zugeordnet sei. Dies könne das Ziel sein, dem man sich beim Bau optischer Geräte zu nähern trachten solle, in wissenschaftlich strengem Sinn könne es aber nur von einer spiegelnden Ebene wirklich erreicht werden. Gibt es im allgemeinen keine zugeordneten Punkte, so kann doch in einem gewissen Sinn von abbildbaren Linien gesprochen werden, wenn diese sich auch nicht Punkt für Punkt entsprechen. Durch jeden Punkt einer Dingfläche verlaufen zwei sich unter einem endlichen Winkel kreuzende Linien, die beide auf zwei Flächen des Bildraumes abgebildet werden, jede auf einer anderen Fläche. Die Richtung dieser Linien und die Lage der Bildfläche werden ange-geben.

Wie so mancher große Geist empfand Gullstrand es offenbar schmerzlich, daß seine Zeit nicht mit ihm zu den Zielen vordrang, die er erreicht hatte und von seiner Wissenschaft berücksichtigt zu wissen 
330

Allvar Gullstrand †.

wünschte. Nur so ist die Schärfe und Bitterkeit zu verstehen, die sich gelegentlich in Schrift und Rede äußert. Daß auch die technische Optik ihre leistungsí'ähigen, im Gerätebau bewährten Verfahrcn nicht aufgegeben hat, mag den Schöpf·er der neuen Lehre enttäuscht haben. Man wird aber bei optischen Werkstätten, in welchen ja die wissenschaftliche Optik sehr wesentlich fußt, zuimal in cliesen wirtschaftlich schweren Zeitläufen, triftige Gründe dafür annehmen dürfen. Um die Vorgänge bei der Lichtbrechung im Menschenauge zu behan-deln, genügen die auf gleichmäßig brechende Mitt el abgeleiteteii Regeln nicht, dazu ist die Beherrschung der ges > chichteten Mittel mit einer von außen nach innen stetig zunehmenden Brechzahl, wie sie in der Linse des Menscbenauges vorliegen, Voraus: etzung. Auch diese Arbeit hat Gullstrand geleistet. Hier sei no·ch der Aufdeckung der eigentümlichen Vorgänge gedacht, die sich bei der Akkommodation fur die Nähe im Linseninneren abspielen, die er als intrakapsulären Akkommodationsmechanismus be-schrieben und 1911 zum Gegenstancl seines Nobelvort rages gemacht hat.

Gullstrand begnügte sich nicht mit clem Wissen, sondern kümmerte sich auch ernstlich um die Ausführung von optischen Geräten. So lag ihm die Verwendung asphärischer Lins en wegen ihrer großen Bedeutung für die optische Abbildung so sehr am Herzen, daß er eingehende Untersuchungen über technische Verfahren zur Herstellung solcher Flächen angestellt und in einer umfangreichen Arbeit veröffentlicht hat. An physiologischen Arbeiten ist noch die über den Farbensinn und die über die Farbe der Netzhautgrubengegend zu nennen. Auch in der Aussprache über die Eínsteinsche Lehre hat sich Gullstrand vernehmen lassen.

E. Einem überragenden Geist war es vergönnt seine Schwingen unge-hemmt in freiem Raum zu hohem Flug zu regen. Die innere Befriedigungzu einem großen Ziel gclangt zu sein blieb ihm nicht vorenthalten. Auchdie äußere Anerkennung seiner Lcistungen hat er erleben dürfen. DieFachwelt hat bewundernd zu ihm aufgeblickt und mit Ehrungen nichtgekargt. 1911 wurcle ihm der Nobelpreis für Meclizin zuerkannt. Diephilosophischen Fakultäten von Upsala Jena und Dublin ernannten ihnehre $\pi$ halber zum Doktor

die schwedische ärztliche Gesellschaft

die eng-lische augenärztliche Gesellschaft

die ärztliche Gesellschaft in Wien

diefinnicche ärztliche Gesellschaft wählten ihn zu ihrem Ehrenmitglied:er wurde Mitglied der preußischen Akademie der Wissenschaften. Fürunsere Deutsche Ophthalmologische Gesellschaft mag es heute ein tröst-licher Gedanke sein daß sie ihn 1928 mit ihrem höchsten Preis mit derGrae†e-Mфázúlle hat ehren und erfreuen dürfen. //. Erggelef. 


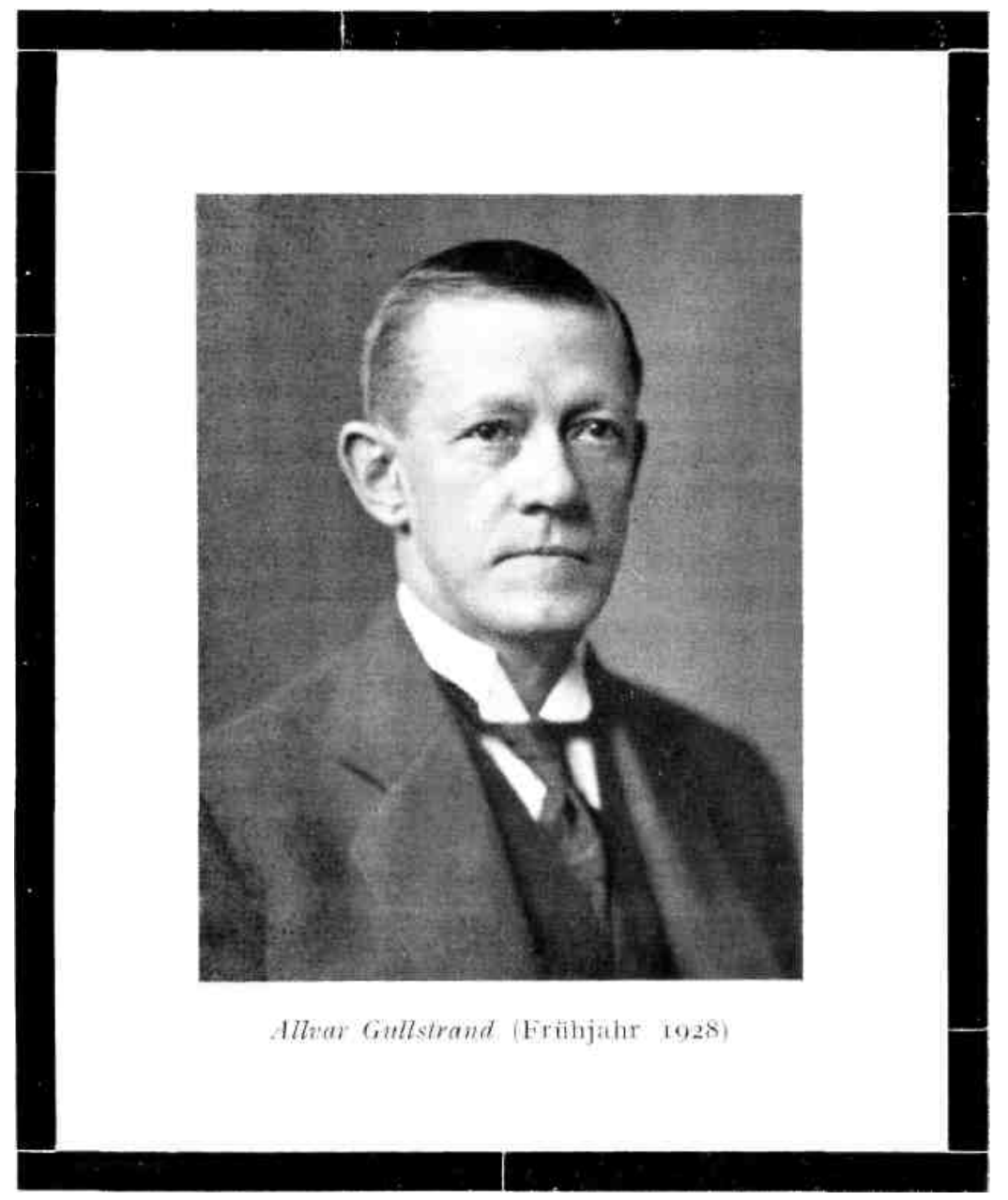

